\title{
Three-dimensional structures of steroids and their protein targets
}

\author{
W. L. Duax, D. Ghosh, V. Pletnev and J. F. Griffin \\ Hauptman-Woodward Medical Research Institute, Inc. 73 High St., Buffalo, NY 14203-1196, USA
}

\begin{abstract}
Steroid receptors and enzymes that control steroid synthesis and metabolism work together to control vital hormonal functions. Crystallographic data on steroids having high affinity for estrogen, progestin. and corticoid receptors suggest that receptor binding is primarily the result of a high level of complementarity between the receptor and the steroidal A-ring. The crystal structures of two steroid dehydrogenase enzymes reveal the location of the active site, the position of the conserved catalytic triad (Tyr-Lys-Ser), a possible mechanism of keto-hydroxyl interconversion and the basis for selectivity. A complex between carbenoxolone and one of the enzymes reveals how the active ingredient in licorice inhibits this enzyme as well as human $11 \beta$-hydroxysteroid dehydrogenase (11 $\beta-H S D)$. The complex reveals that the inhibitor fills the substrate binding pocket, displaces the cofactor and accepts a hydrogen bond from a tyrosine residue, one of less than 20 strictly conserved residues in the family of enzymes, explaining how ingestion of licorice can produce hypertension.
\end{abstract}

Introduction.

Steroid hormones play a vital role in a wide variety of essential physiological processes including cell growth, sexual development, maintenance of salt balance and sugar metabolism. They have also been implicated in responses to stress and sexual imprinting of the fetal brain. Abnormalities in steroid hormone synthesis, metabolism and receptor interaction contribute to a variety of diseases. Synthetic steroids are used extensively in fertility control, as anti-inflammatory agents and immunosuppressants. Antihormones that interfere with the synthesis, metabolism and receptor binding of steroids are useful in some forms of chemotherapy. A careful examination of the molecular structures and three-dimensional shapes of the hormones, antihormones, chemicals and drugs that compete for a common binding site on a specific receptor, binding protein or metabolizing enzyme can provide information on structural features that influence hormonal response.

Crystallographic data on over 1000 steroids $(1,2)$ provide information concerning preferred conformations, relative stabilities and substituent influence on the interactive potential of steroid hormones and analogs. Systematic conformational analysis of subsets of these data having common structural features suggests that steroids crystallize in global minimum energy conformations or local minimum energy conformations that are less than two $\mathrm{kcal} \mathrm{mol}^{-1}$ above the global minimum (3-5). If the receptorbound steroid is also in its minimum energy conformation, then it should be possible to compare the crystallographically observed structures of a series of steroids that compete for a specific binding site and determine the structural features that are essential for binding, the relative location of functional groups that interact with the receptor, how tight a fit exists between the steroid and the receptor, and to what extent the binding site of the receptor protein is flexible. This paper describes a proposed empirical model for steroid receptor binding based on structure-activity comparisons and a description of efforts to model the steroid binding pocket in a family of hydroxysteroid dehydrogenase enzymes.

\section{Steroid Structure, Receptor Binding and Hormone Action}

Examination of the structures of compounds having high affinity for estrogen, progestin, and corticoid receptors led to the suggestion that steroid-receptor binding is primarily the result of interactions between the receptor and the steroidal A ring (5-8). An illustration of the proposed fit between the phenolic A ring present in almost all compounds exhibiting estrogenic activity and a binding site on the receptor is illustrated in Figure 1. Activity of estrogens, progestins, and corticoids might be controlled by the $D$ ring. Possible mechanisms include (1) inducing or stabilizing an essential conformational state in the receptor (allostery), (2) influencing the aggregation state of the receptor, or (3) participating in a direct interaction

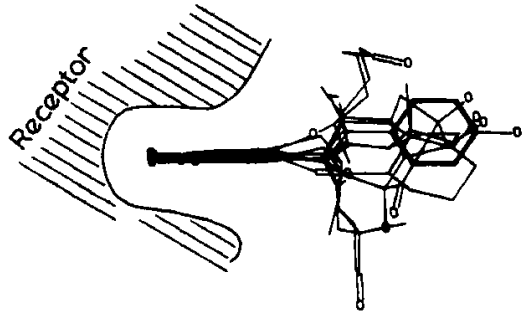

Fig. 1. The superposition of the phenol rings of six molecules that bind to the estrogen receptor suggests that variability in D-ring orientation is compatible with receptor binding and some degree of activity. 
with DNA or chromatin (9). Antagonists that compete for these receptor sites appear to have the A-ring composition and conformation necessary for receptor binding but lack the D-ring conformational features and functional groups that induce or stabilize subsequent receptor functions (10). Antagonists also include compounds with A-ring conformations appropriate for binding but have other structural features that interfere with subsequent receptor functions essential to activity. In contrast, androgen receptor binding data (11) and molecular modeling studies suggest that for androgens a D-ring binding/A-ring acting model is more appropriate.

\section{Steroid-Protein Interactions and Binding}

Information concerning the molecular details of steroid-protein interactions can be obtained from X-ray analysis of crystalline complexes of proteins with appropriate steroid agonists and antagonists, and from $\mathrm{X}$-ray studies of model complexes of steroids with amino acids. The structure of uteroglobin, a progesterone-binding protein (12) provides a useful model for other steroid binding proteins, although no steroid was present in its presumed binding pocket. Quantities of pure stable, intact steroid receptor proteins, sufficient to allow single crystal $x$-ray analysis are not yet available; however, the crystal structures of complexes between the DNA-binding domain of the glucocorticoid receptor and specific recognition elements from DNA have been reported (13). Crystal structures of three enzymes that have steroids as their primary substrate have been reported. The steroid-binding site has been tentatively identified in one of these by imaging a bound inhibitor (14) and inferred in the others on biochemical grounds $(15,16)$. The rest of the paper will be concerned with the mechanism of action of these enzymes.

\section{Licorice, Ulcers, High Blood Pressure and Short Chain Dehydrogenase Enzymes}

Studies of three-dimensional structures of a family of dehydrogenase enzymes that influence mammalian reproduction, hypertension, neoplasia and digestion can reveal the mechanisms by which these enzymes control circulating levels of steroids and other hormones and permit the design of ligands that will have selectivity of action. One member of the family controls cortisol/cortisone balance and influences hypertension (17), another controls estradiol/estrone balance and influences growth of certain tumors (18) and a third controls prostaglandin metabolism and is implicated in development of ulcers (19). Although these enzymes have different substrate specificities they can be inhibited by common agents. Of particular interest is the inhibition of several members of the family by an active ingredient in licorice, glycyrnhizic acid and its metabolite carbenoxolone (20). Licorice has a therapeutic effect on digestion reputedly via inhibition of prostaglandin dehydrogenase but excess consumption of licorice is dangerous for people taking medication for hypertension. We have determined the crystal structures of various forms of two members of the short-chain dehydrogenase family, human estrogenic $17 \beta$-hydroxysteroid dehydrogenase (17 $\beta$-HSD) (21) and bacterial $3 \alpha, 20 \beta$-hydroxysteroid dehydrogenase $(3 \alpha, 20 \beta$-HSD) $(22,23)$. The threedimensional structures reveal the position of the conserved catalytic triad (Tyr-Lys-Ser) in the active site, a possible mechanism of keto-hydroxyl interconversion, the mechanism of inhibition by carbenoxolone, and the basis for $17 \beta-\mathrm{HSD}$ selectivity.

\section{$3 \alpha .20 \beta-H y d r o x y s t e r o i d$ Dehydrogenase}

Bacterial $3 \alpha, 20 \beta$-hydroxysteroid dehydrogenase reversibly oxidizes the $3 \alpha$ and $20 \beta$ hydroxyl groups of androstanes and pregnanes. It was the first short-chain dehydrogenase to be studied by $\mathrm{X}$-ray crystallography. The asymmetric unit of the crystal contains four monomers, each with 253 amino acid residues, 38 water molecules, and 176 cofactor atoms belonging to four NAD molecules - one for each subunit. The three-dimensional structure of the monomer of $3 \alpha, 20 \beta$-HSD and the observed location of the cofactor is illustrated in Figure 2 and the organization of the tetramer illustrated in Figure 3.

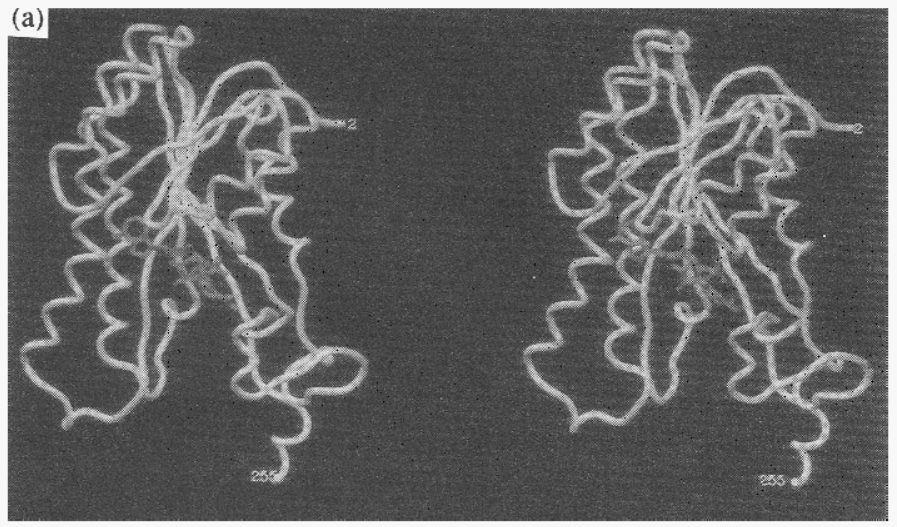

Fig. 2. (a)Stereoview or one subunit of the tetramer of $3 \alpha, 20 \beta-$ HSD shown as an $\alpha$-carbon atom trace. The view is nearly along the edge of the central $\beta$-sheet. The NAD molecule is shown. 


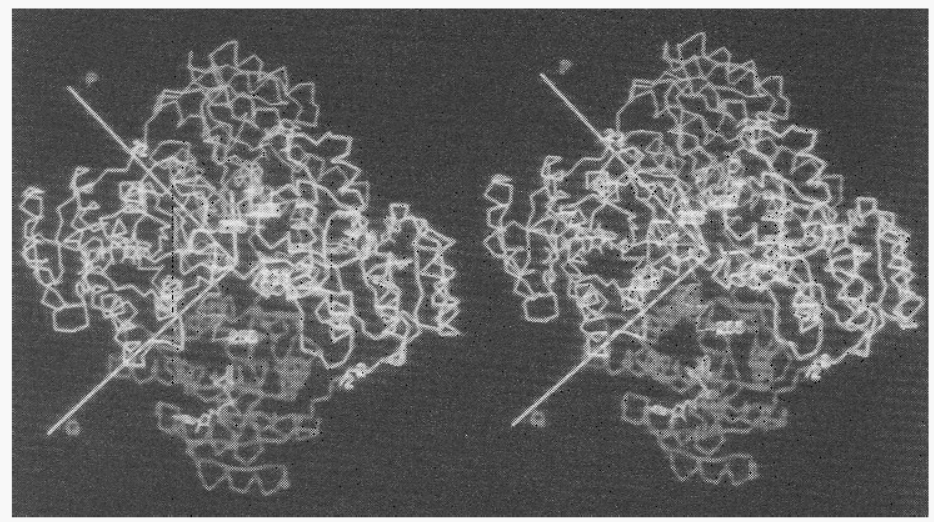

Fig. 3. A stereographic diagram of the tetrameric $3 \alpha, 20 \beta$-HSD. The monomers are drawn as $\alpha$-carbon traces of the polypeptide chains. Subunits at the top and bottom of the picture are shaded to enhance contrast between A, B, C and D subunits. The non-crystallographic 222 symmetry elements are shown as $P, Q$, and $R$ axes. Four NAD molecules are also shown.

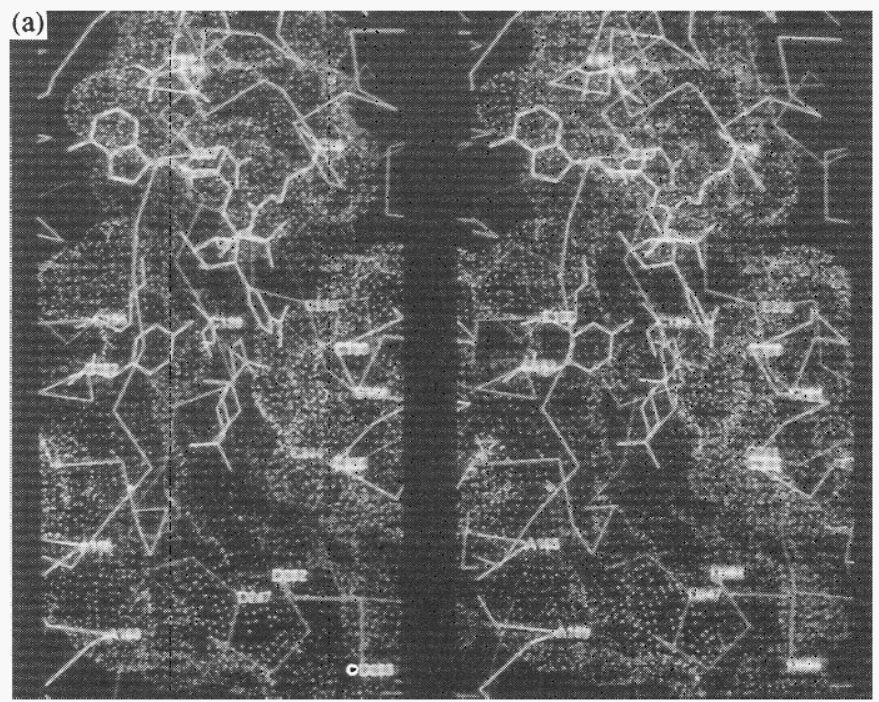

(b)
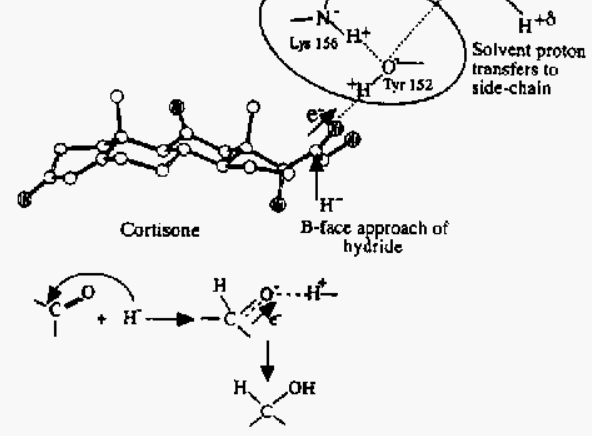

Fig. 4. The crystallographically observed position of the NAD cofactor and residues $\operatorname{Thr} 12, \operatorname{Arg} 16$, Asn87, Ser139, Tyr 152 and Lys 156 in the putative active site of $3 \alpha, 20 \beta-H S D$ are shown in (a). The dotted Connolly surface of the protein defines an accessible channel to the conserved residue Tyr152 and Lys156. A cortisol molecule can be modeled in the site without disrupting the observed side chain conformation or piercing the Connolly surfaces. The distance between the Tyr 152 hydroxyl oxygen and 20 -keto oxygen is about $2.0 \AA$. The 20 -keto oxygen is also within hydrogen bonding distance $(-2.6 \AA)$ of the Ser 139 hydroxyl. Carbon-20 of the steroid is $2.0 \AA$ from the $C 4$ position of the nicotinamide ring. A possible mechanism by which the residues may mediate stereospecific hydride transfer and proton relay processes during the biochemical 20-keto to 20ß-hydroxyl conversion is shown in (b).

The catalytic site contains residues Tyr 152 and Lys 156 . These two amino acids are strictly conserved in the short-chain dehydrogenase superfamily (24). Modeling studies with a cortisone molecule in the catalytic site (Figure 4) suggest that the Tyr152, and Lys 156 side chains promote electrophilic attack on the $\left(\mathrm{C}_{20}-\mathrm{O}\right)$ carbonyl oxygen atom, thus enabling the carbon atom to accept a hydride from the reduced cofactor. The Ser 139, conserved in steroid dehydrogenases could be involved in substrate binding or in some unknown function.

\section{Carbenoxolone Complex of $3 \alpha, 20 \beta-H S D$}

We demonstrated that glycyrrhizic acid and carbenoxolone are potent inhibitors of $3 \alpha, 20 \beta-H S D(20)$ and prepared single crystals of a complex between carbenoxolone and $3 \alpha, 20 \beta-H S D$. The three-dimensional structure of the enzyme-carbenoxolone complex was determined and refined at $2.2 \AA$ resolution to a crystallographic $R$-factor of $19.4 \%$. The hemisuccinate side chain of carbenoxolone makes a hydrogen bond with the hydroxyl group of the conserved residue Tyr152 and occupies the position of the nicotinamide ring of the cofactor (Figure 5). The inhibitor binds at the catalytic site in a mode much like the previously proposed mode of binding of the substrate cortisone. 


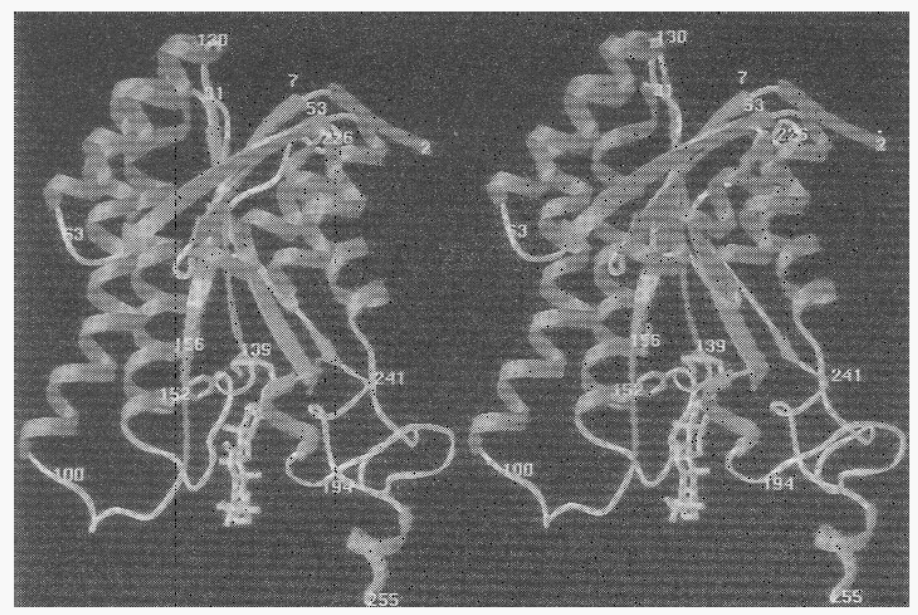

Fig. 5. A Stereoview of the crystallographically observed complex of $3 \alpha, 20 \beta$-HSD and carbenoxolone. a potent inhibitor of the enzyme. The hemisuccinate chain of carbenoxolone is surrounded by the conserved catalytic residues Ser 139, Lys156, and Tyr 152 and is hydrogen bonded to the latter.

This structure presents the first direct evidence of binding of a substrate-like moiety in the catalytic site of a member of this family of enzymes. The structure of the complex of $3 \alpha, 20 \beta$-HSD with the active ingredient in licorice, reveals that the steroid molecule binds in the region previously identified as the catalytic site, thereby preventing substrate binding. Carbenoxolone also competes effectively with the binding of the essential cofactor nicotinamide adenine dinucleotide. The binding interactions include the formation of a specific hydrogen bond with the invariant Tyr 152. Together with the site-directed mutagenesis study that showed tyrosine to be essential for catalytic activity (25-27), this result suggests that Tyr 152 could interact with steroidal substrates in a similar fashion. Analogous binding of carbenoxolone to human renal $11 \beta$-HSD is reported to be a contributing factor in licorice-induced hypertension. Detailed information on the structure of holo, apo, and inhibited forms of the short-chain dehydrogenase family of enzymes will be critical to determining their mechanisms of action and the design of selective and specific inhibitors. Such inhibitors could have direct therapeutic applications as drugs with reduced side effects for the treatment of certain hormone related diseases.

\section{7ß-Hydroxysteroid Dehydrogenase}

Because human estrogenic $17 \beta$-hydroxysteroid dehydrogenase (type I 17ß-HSD) catalyzes the last step in the biosynthesis of $17 \beta$-estradiol from the less potent estrogen, estrone, it is an attractive target for the design of inhibitors of estrogen production and tumor growth. This human enzyme shares less than $15 \%$ sequence identity with a bacterial $3 \alpha, 20 \beta$-HSD. The amino acid sequence of $17 \beta$-HSD also differs from that of bacterial $3 \alpha, 20 \beta-H S D$ by insertions of 11 and 14 residues and the addition of 52 more residues at the $\mathrm{C}$ terminus. The $2.20 \AA$ resolution structure of type I 17 $\beta$-HSD, the first mammalian steroidogenic enzyme studied by X-ray crystallographic techniques, reveals a fold characteristic of the short-chain dehydrogenases. The active site contains a Tyr-X-X-X-Lys sequence (where $X$ is any amino acid) and a serine residue, features that are conserved in short-chain steroid dehydrogenases. The structure also contains three $\alpha$-helices and a helix-turn-helix motif, not observed in short-chain dehydrogenase structures reported previously.

Our results demonstrate that despite only about $15 \%$ sequence identity and the presence of two major insertions and additional residues at the $C$ terminus, $17 \beta$-HSD has the same overall fold as bacterial $3 \alpha, 20 \beta-H S D$ and mammalian DHPR. The additional residues in 17 $\beta$-HSD form two helix-turn-helix motifs, and these constitute a 'substrate-recognition domain' bordering the active site at the end of the steroid-binding cavity opposite the cofactor-binding site and the catalytic residues. The apparent amphiphilic nature of the additional helices and their :opological location are consistent with the observed association of the enzyme with microsomal membranes. Modeling $17 \beta$-estradiol at the active site suggests that the 3-hydroxyl end of the substrate, which is at the end of the molecule opposite to where the oxidoreductive reactions occur, fits into a cavity in this domain and forms a specific hydrogen bond with a histidine side chain (Figure 7). Comparable substrate-recognition features are not present in the active site of the bacterial enzyme, allowing it to process a greater range of substrates. The proposed transition state of the enzyme is consistent with the relative locations of the recognition histidine (a histidine side chain that makes a specific hydrogen bond with the non-reactive 3-hydroxyl end of the substrate) and the catalytic Tyr-Ser-Lys triad in the active site (Figure 8). The model of the active site of 17B-HSD derived from the present structure analysis provides a template for the design of specific inhibitors as drugs for breast 


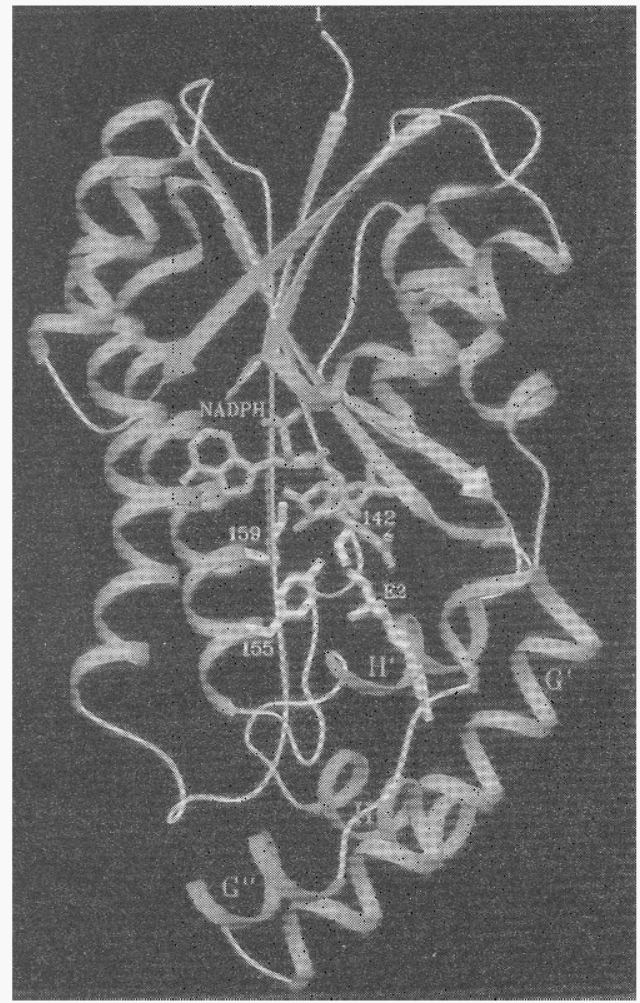

Fig. 6. In the ribbon diagram of the crystallographically observed structure of $17 \beta-H S D$, the conserved Ser 142, Tyr 156 and Lys 159 are illustrated. The cofactor position is modeled on the basis of similarity to $3 \alpha, 20 \beta$-HSD and steroid modeling in the solvent filled position. The helical insertions in 17 $\beta$-HSD that help define substrate specificity relative to other short chain dehydrogen enzymes $\left(\alpha G^{\prime \prime}, \alpha G^{\prime}, \alpha H^{\prime}\right.$ and $\alpha \mathrm{H})$ are at the lower right.

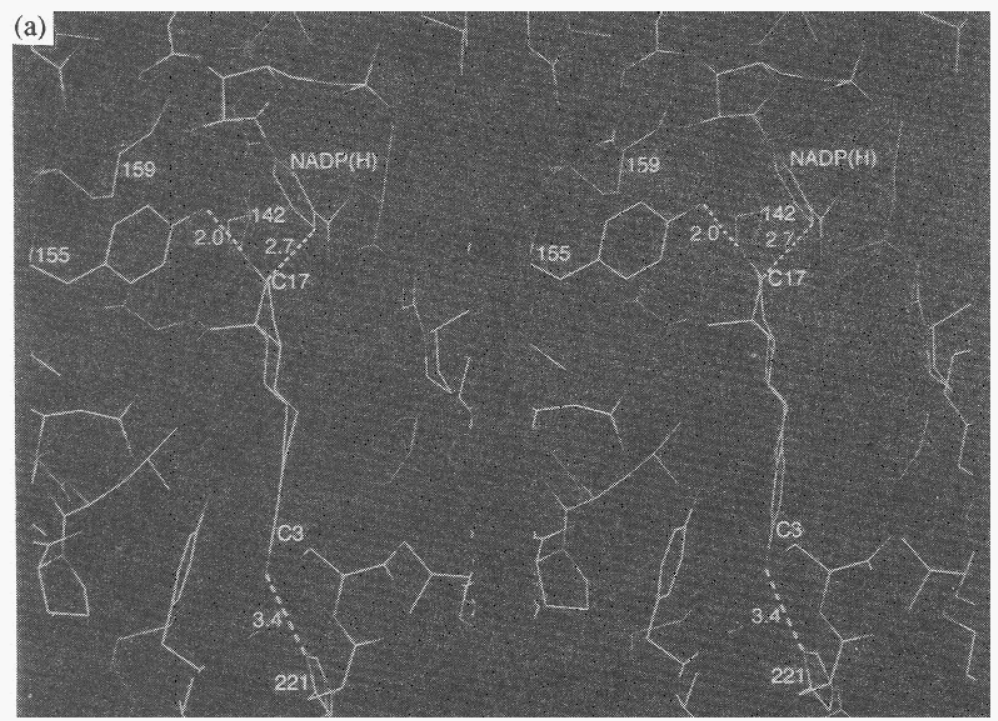

Fig. 7. (a) Stereoview of the atomic model of the proposed transition state of estrone to estradiol interconversion. All atomic positions of the protein were observed and those of the cofactor and the steroid molecule that were derived from modeling. The $\beta$-face of the steroid is oriented towards Tyr 155 and the pro-S hydride from the $\mathrm{C} 4$ atom of the nicotinamide ring approaches close to $\mathrm{C} 17$ of $17 \beta$ estradiol. (Figure prepared using the program CHAIN.) 


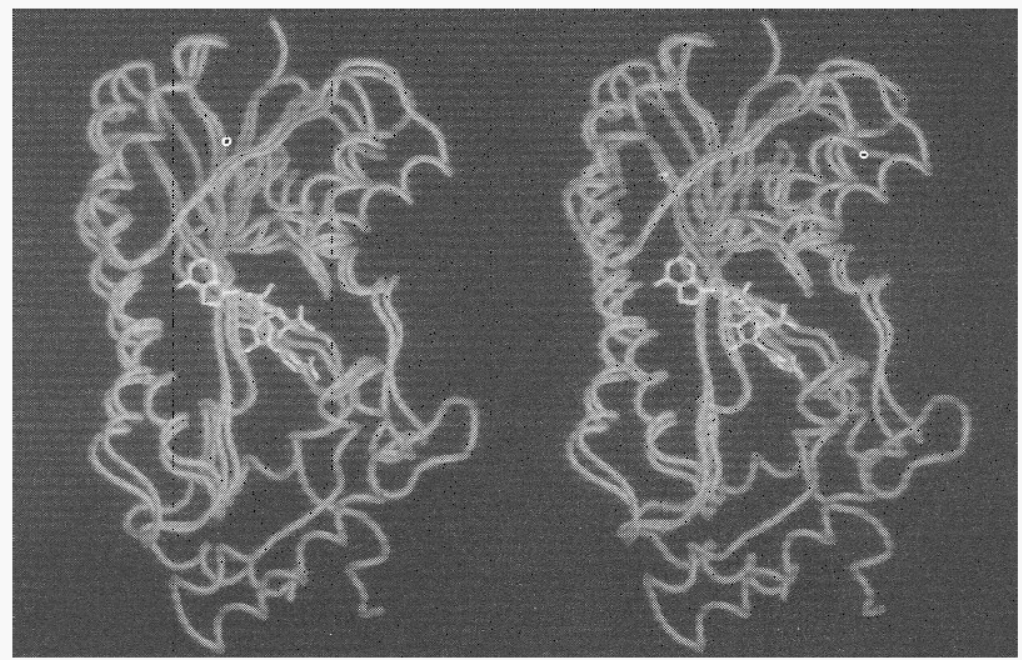

Fig. 8. A stereodiagram of superimposed $\mathrm{C} \alpha$ chains of bacterial $3 \alpha, 20 \beta-\mathrm{HSD}$ and human estrogenic $17 \beta$-HSD. The superposition was achieved by least-squares fitting $20 \mathrm{C} \alpha$ atoms that are at similar positions in both structures. The cofactor, NAD bound to $3 \alpha, 20 \beta-\mathrm{HSD}$ is also shown. (Figure prepared using the program SETOR.)

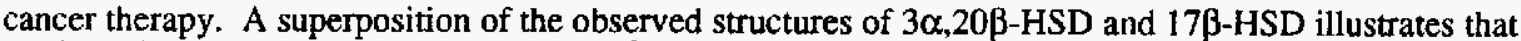
the insertion and additional residues in $17 \beta-H S D$ are clustered at one end of the substrate binding site away from the cofactor binding site. The insertion and specific residues in the binding of $17 \beta-\mathrm{HSD}$ account for the specificity of action of the enzyme. In addition the insertions prohibit inhibition of this member of the short chain dehydrogen family by carbenoxolone and glyccyrrhizic acid, demonstrating that selective inhibitors of individual members of the short chain dehydrogen family is favorable.

Acknowledgements

Research supported in part by the National Institute of Diabetes, and Digestive and Kidney Diseases Grant No. DK26546.

\section{References}

1. W.L. Duax and D.A. Norton. Atlas of Steroid Structure, Vol. I, Plenum, New York (1975).

2. J.F. Griffin et al. Atlas of Steroid Structure, Vol. 2, Plenum, New York (1984).

3. W.L. Duax et al. Topics in Stereochemistry, pp. 271-383, Wiley-Interscience, New York (1976).

4. W.L. Duax et al. J. Am. Chem. Soc. 103, 6705-6712 (1981).

5. W.L. Duax et al. Intramolecular Dynamics, pp. 505-524, D. Reidel, Dordrecht (1982).

6. W.L. Duax et al. J. Tox. Env. Health 4, 205 (1978).

7. W.L. Duax and J.F. Griffin. J. Ster. Biochem. 27, 271 (1987).

8. W.L. Duax et al. J. Ster. Biochem. 15, 41 (1981).

9. W.L. Duax and J.F. Griffin. Advances in Drug Research, Vol. 18, pp. 115-138, Academic Press, New York (1989).

10. W.L. Duax et al. J. Ster. Biochem. 31, 481 (1988),

11. J. Kirchhoff et al. J. Ster. Biochem. 10, 487-497 (1979).

12. D. Ghosh et al. Proc. Natl. Acd. Sci. USA 88, $10064-10068$ (1991).

13. I. Morize et al. J. Mol. Biol. 194, 725 (1987).

14. B.F. Luisi et al. Nature 352, 497 (1991).

15. E.M. Westbrook et al. J. Biol. Chem. 259, 9096 (1984).

16. A. Vrielink et al. J. Mol. Biol. 219, 533-550 (1991).

17. Z. Krozowski. Molecular and Cellular Endocrinology 84, 25-31 (1992).

18. L.L. Engel and E.V. Groman. Recent Prog. Horm. Res. 30, 139-169 (1974).

19. M.E. Baker. Prostaglandins 42, 391-410 (1991).

20. D. Ghosh et al. J. Steroid Biochem. Mol. Biol. 42, 849-853 (1992).

21. D. Ghosh et al. Structure 3, 503-513 (1995).

22. D. Ghosh et al. Structure 2, 629-640 (1994).

23. D. Ghosh et al. Structure 2, 973-980 (1994).

24. H. Jörnvall et al. Biochemistry 34, 6003-6013 (1995).

25. Z. Chen et al. Biochemistry 32, 3342-3346 (1993).

26. C.M. Ensor and H.-H. Tai. Biochem. Biophys. Res. Commun. 176, 840-845 (1991).

27. J. Obeid and P.C. White. Biochem. Biophys. Res. Commun. 188, 222-227 (1992). 